\title{
EXAMPLES OF EXTREME INTRADAY VARIABILITY
}

\author{
LUCYNA KEDZIORA-CHUDCZER ${ }^{1,2}$, DAVID L. JAUNCEY ${ }^{2}$, \\ MARK A. WIERINGA ${ }^{2}$, ANASTASIOS K. TZIOUMIS ${ }^{2}$ and HAYLEY E. BIGNALL ${ }^{3}$ \\ ${ }^{1}$ Anglo-Australian Observatory \\ ${ }^{2}$ Australia Telescope National Facility, Australia \\ E-mail: \{lkedzior;djauncey;mwiering;atzioum\}@atnf.csiro.au \\ ${ }^{3}$ University of Adelaide, Australia; E-mail: hbignall@atnf.csiro.au
}

\begin{abstract}
We present results of the ATCA IDV Survey of southern extragalactic radio sources. We discuss briefly the properties of the 22 new intraday variable sources discovered in the Survey. The follow-up observations of a few extreme examples of strong intraday variability are presented. We find that the characteristics of the total flux density fluctuations at different wavelengths are consistent with intersteller scintillations (ISS) of the microarcsecondsize soorten components. However, the scintillating components of a few extreme IDVs are characterized by the brightness temperatures far exceeding the $T_{B}=10^{12} \mathrm{~K}$ limit. The relativistic beaming invoked in such sources would require Doppler factors up to as high as $\delta \sim 10^{3}$.
\end{abstract}

\section{Introduction}

More than a decade from the discovery of intraday variability (IDV) in extragalactic radio sources (Witzet et al., 1986) its origin is still debated. The interpretations of IDV fell into two categories, those intrinsic to the source and propagation effects (Wagner and Witzel, 1995). Any model of the intrinsic IDV puts stringent limits on the size of fluctuating sources ( $<1$ light day), which leads to excessive brightness temperatures, $T_{B} \gg 10^{12} \mathrm{~K}$. Such high brightness temperatures are usually explained in terms of relativistic beaming.

Two frequently considered extrinsic models are microlensing of the source by the stars in the foreground galaxies, and scintillation (ISS) in the interstellar medium of our Galaxy.

The ATCA IDV Survey was aimed at identifying a sample of new IDV soorten, and studying their properties and statistics. We selected 118 flat spectrum, compact soorten from the PTI Survey (Duncan et al., 1993). The sample was observed with the AT Compact Array at four frequencies: $8.6,4.8,2.5$ and $1.4 \mathrm{GHz}$ during two campaigns in May and August 1994 (Kedziora-Chudczer et al., 2000). 


\section{Results of the ATCA IDV Survey}

We discovered 22 new IDV sources, which show significant total flux density fluctuations on the timescale of 96 hours. Variability appears to be strongest at 4.8 and $2.4 \mathrm{GHz}$. We measured flux densities in all four Stokes parameters and we found 15 sources which show IDV in the linear polarization. The timescale of variability can be as short as a few hours (e.g. PKS 0405-385) but it also extends beyond 1 day in a few sources (e.g. PKS 1144-379 and PKS 1034-293). The timescales typically increase with the decreasing frequency.

We find that IDV is episodic in two thirds of the sample. Only six sources (PKS 0808+019, PKS 1034-293, PKS 1048-313, PKS 1144-379, PKS 1519-273, PKS 1556-245, PKS 1622-297) showed IDV in both observing sessions. However $75 \%$ of IDV sources showed longer term variations on a timescale of months. The comparison of Parkes Telescope flux density measurements and our data shows that all flatspectrum radio sources are variable over a timescale of decades at 5 and $2.7 \mathrm{GHz}$.

We observe that inverted spectrum radio sources dominated by a flux density of the compact components, are most likely to show IDV (39\% of all inverted spectrum sources in the sample). Our IDV sample consists of 6 BL Lacs (50\% of all BL Lacs in the total sample) and 16 quasars (20\% of all quasars in the whole sample). We find mostly low-redshift objects among our IDV sources (KedzioraChudczer et al., 2000).

\section{The Long Term Monitoring of PKS 0405-385}

The most intriguing and extreme IDV source discovered in the ATCA IDV Survey is a quasar, PKS 0405-385 at $z=1.285$. The episodic nature of IDV in this source is visible in Figure 1. The long term lightcurve is a composite of continuous observations over days, and the weekly monitoring of the source over $\sim 20$ minutes duration each time. The IDV Survey data are also shown (2449300JD, 2449491JD and 2449592JD). The well sampled set of data startmg on 2450242JD reveals strong and rapid IDV (Kedziora-Chudczer et al., 1997), which ceased after two months. After 2.5 years of a slow flux density evolution the rapid variability resumed in Nov 1998 (2451143JD) and lasted for 4.5 months. In Kedziora-Chudezer et al. (1997) we proposed that the episodic IDV can be explained by the interstellar scintillation (ISS) of the compact source component, which contained $15 \%$ of the total flux density. Scintillations disappeared due to the increase in size of this component. This interpretation fits well with the appearance of a flare visible after a period of IDV in the long term lightcurve in Figure 1. However a subsequent outburst of the IDV observed in 1998 was not followed by an increase in the average total flux density. The interpretation of episodic IDV in this source is still unclear. 


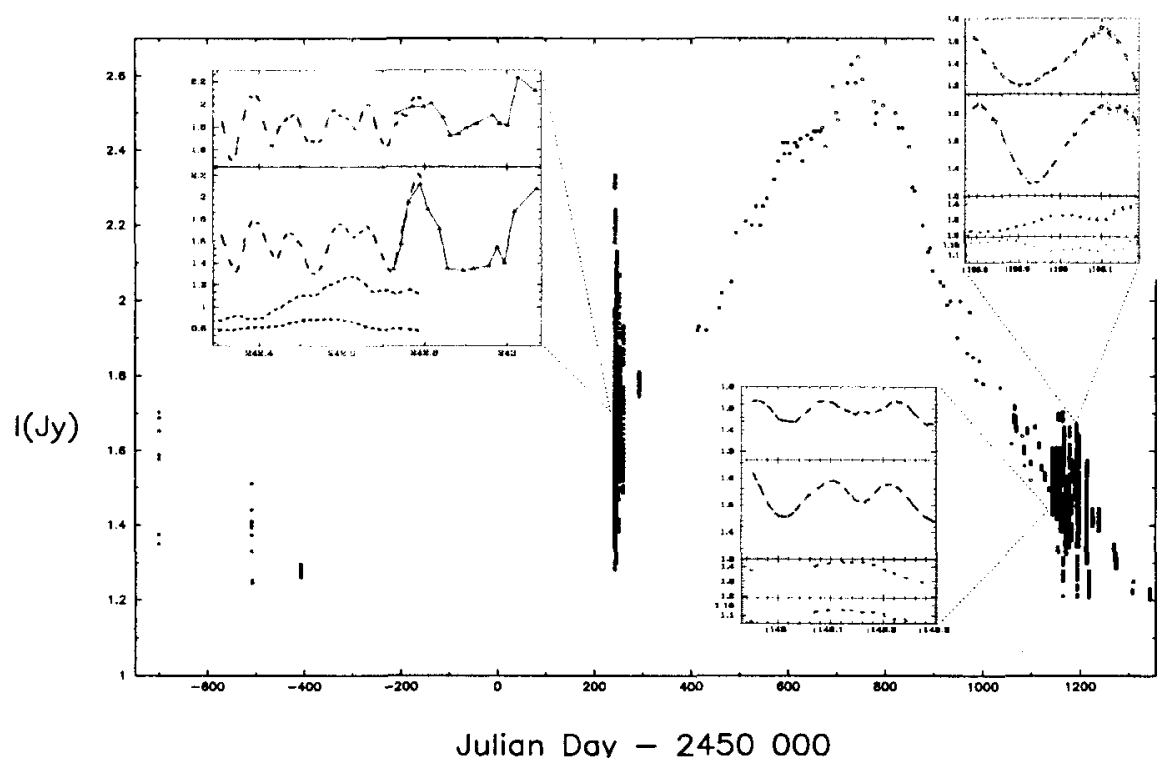

Figure 1. Long term monitoring of PKS 0405-385 with the ATCA at $4.8 \mathrm{GHz}$. The inserted boxes contain continuous measurements of flux densities over 12 to 24 hours during the IDV episodes at four frequenties: $8.6,4.8,2.5$ and $1.4 \mathrm{GHz}$ (from top to bottom).

\section{Simultaneous Observations of 4 Southern IDV Sources}

We carried out a number of monitoring observations of a few of the strongest IDV sources discovered in the ATCA IDV Survey. Figure 2 shows six days of the total flux density data at different frequenties. The range of IDV timescales is clearly present in these data. At high frequenties, the BL Lac source, PKS 1144-379, exhibits hourly flux density fluctuations on top of stronger variability with a daily timescale, as compared with PKS 1519-273 which seems to vary with only one typical timescale of a few hours. PKS 1034-293 and PKS 1622-297 are examples of IDV sources with progressively longer timescales. In Figures 2(a) and (b) we plot the 4-frequency lightcurves for PKS 1144-379 and PKS 1519-273 to illustrate a characteristic increase of IDV timescale with decreasing frequency.

\section{Summary}

The observed variability can be explained in terms of scintillation theory. In the case of intrinsic IDV, the source sizes implied by the variability timescales (lighthours to lightdays) are small enough that scintillations must contribute to flux density fluctuations. The detailed models of the ISS induced intraday flux density fluctuations for the most extreme IDVs: PKS 0405-385 (Kedsiora-Chudczer et al., 1997) and PKS 1519-273 (Macquart et al., 2000) suggest that the inferred 


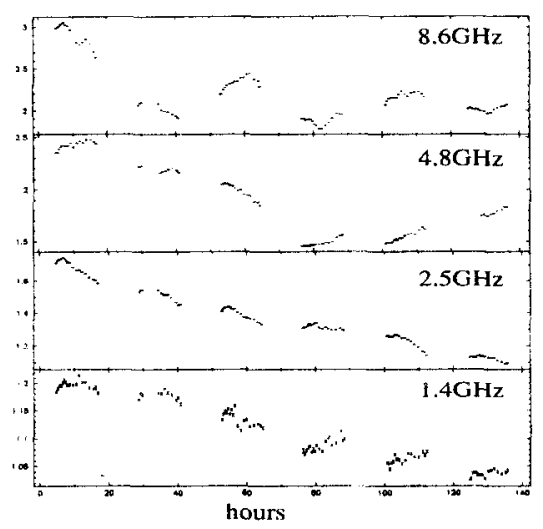

(a) PKS 1 144-379

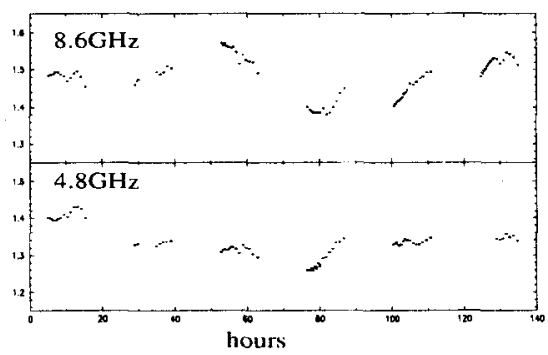

(c) PKS1034-293

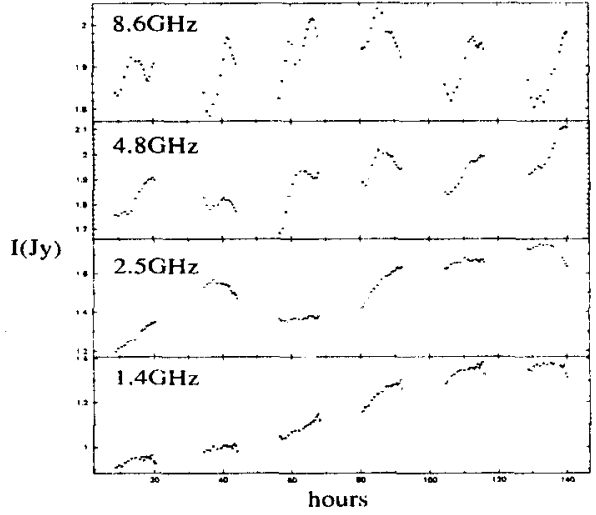

(b) PKS 1519-273

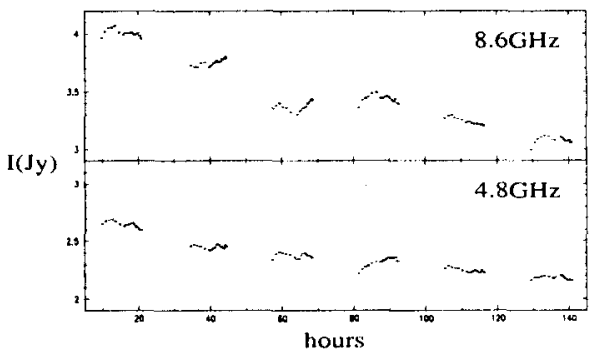

(d) PKS1622-297

Figure 2. Monitoring of four IDV sources over six days in May 1999. The scatter in the $1.4 \mathrm{GHz}$. lightcurve of PKS 1144-379 arises due to sidelobes of the confusing source in the beam of the ATCA.

brightness temperature of the scintillating components is $T_{B}>10^{14} \mathrm{~K}$. Such high brightness temperatures can be understood as an effect of relativistic beaming, if one is prepared to accept Doppler factors higher than $\delta \sim 10^{2}$. The problem of high brightness temperature can be addressed in terms of coherent emission mechanisms. However, a convincing model of coherent emission in IDV sources has yet to be developed.

\section{Acknowledgements}

The Australia Telescope is funded by the Commonwealth Government for operatien as a national facility by the CSIRO.

\section{References}

Duncan, R.A., White, G.L., Wark, R., Reynolds, J.E., Jauncey, D.L., Norris, R.P. and Taaffe L.: 1993 , Proc. ASA 10, 310P. 
Kedziora-Chudczer, L.L., Jauncey, D.L., Wieringa, M.H., Reynolds, J.E. and Tzioumis, A.K.: 2000, MNRAS, submitted.

Kedziora-Chudczer, L.L., Jauncey, D.L., Wicringa, M.H., Walker, M.A., Nicolson, G.D., Reynolds, J.E. and Tzioumis, A.K.: 1997 ApJL 490, L9.

Macquart, J.-P., Kedziora-Chudezer, L., Jauncey, D.L. and Rayner, D.P.: 2000, to appear in ApJ 538. Rickett, B.J., Quirrenbach, A., Wegner, R., Krichbaum, T.P. and Witzel, A.: 1995, A\&A 293, 479P. Wagner, S.J. and Witzel, A.: 1995, ARA\&A 33, 163P.

Witzel, A., Heeschen, D.S., Schalinski, C.J. and Krichbaum, T.P.: 1986, Mitt. Astron. Ges. 65, 239P. 